\title{
Atitudes e percepções de gestores ambientais da Zona Costeira de SP frente às mudanças climáticas
}

\author{
Attitudes and perceptions of environmental managers in relation to climate \\ change in the Coastal Region of the State of São Paulo, Brazil \\ Anna Paula Peres ${ }^{1}$ (D), Nathalia Clyo Rizzo de Freitas Neves ${ }^{1}$ (D), Renzo Taddei $^{2}$ (D) \\ ${ }^{1}$ Instituto do Mar, Bacharelado Interdisciplinar em Ciência e Tecnologia do Mar, Universidade Federal de São Paulo \\ (UNIFESP), Santos (SP), Brasil. E-mails: peres.anna@outlook.com, nathaliaclyo@gmail.com \\ ${ }^{2}$ Instituto do Mar, Programa de Pós-graduação Interdisciplinar em Ciência e Tecnologia do Mar, Universidade Federal de São \\ Paulo (UNIFESP), Santos (SP), Brasil. E-mail: renzo.taddei@unifesp.br
}

\begin{abstract}
Como citar: Peres, A. P., Neves, N. C. R. F., \& Taddei, R. (2020). Atitudes e percepções de gestores ambientais da Zona Costeira de SP frente às mudanças climáticas. Revista de Economia e Sociologia Rural, 58(2), e191793. https://doi.org/10.1590/18069479.2020.191793
\end{abstract}

Resumo: A zona costeira do estado de São Paulo é uma região considerada altamente suscetível aos efeitos das mudanças climáticas. Tal situação é agravada pelo fato de a região concentrar atividades econômicas de relevância nacional e grande densidade populacional, em adição a reservas ambientais e áreas de proteção legal. O presente estudo tem como objetivo analisar as percepções, conhecimentos e atitudes de gestores públicos sobre os efeitos das mudanças climáticas na região costeira do estado, além de avaliar o tipo de informação que possuem e usam (ou pretendem utilizar) na elaboração de planos de adaptação. A pesquisa foi realizada a partir da aplicação de questionário via plataforma online SurveyGizmo. Os resultados obtidos documentam níveis crescentes de preocupação, por parte dos gestores ambientais, com os impactos das mudanças climáticas; ao mesmo tempo, evidenciam a ausência de atividade efetiva de elaboração de políticas públicas que visem mitigar ou se adaptar aos impactos esperados.

Palavras-chave: mudanças climáticas, zona costeira, gestão ambiental, mitigação e adaptação.

Abstract: Previous studies have shown that the coastal zone of the State of São Paulo, Brazil, is considered highly susceptible to the impacts of climate change. This scenario is complicated by the presence of nationally relevant economic activities, large population and legally protected ecosystems. The present study aims to analyze perceptions, knowledge and attitudes of environmental managers about the effects of climate change on coastal regions, as well as to evaluate the type of information that is used (or planned to use) in the construction of effective adaption plans. This research was carried out through the application of a survey using the online platform SurveyGizmo. The results document growing levels of preoccupation among environmental managers about the impacts of climatic changes; at the same time, they make evident the absence in effective activity in the creation of public policy addressed to the mitigation or adaptation to the expected impacts.

Keywords: climate change, coast zone, environmental management, mitigation and adaptation

\section{INTRODUÇÃO ${ }^{1}$}

As regiões costeiras concentram, em nível planetário, grande parte da população e das atividades econômicas de diversos setores; além disso, são importantes em termos ecológicos (Holligan \& Deboois, 1993; Turner et al., 1996; Sachs et al., 2001). A somatória destes fatores torna tais regiões especialmente vulneráveis aos impactos das mudanças climáticas (Klein \& Nicholls, 1999). O aumento do nível do mar e seus impactos em cidades

${ }^{1} \mathrm{~A}$ pesquisa contou com financiamento do Center for Research on Environmental Decisions/National Science Foundation (SES-0345840 e SES-0951516) e do Inter-American Institute for Global Change Research (CRN-3035) 
costeiras e o deslizamento de encostas são exemplos de como as mudanças climáticas exacerbam as vulnerabilidades já existentes, agravando problemas socioeconômicos, logísticos e desafios políticos (Frumkin et al., 2008; UNESCO, 2013).

Neste contexto, a zona costeira do estado de São Paulo mostra-se uma região altamente suscetível aos impactos de alterações ambientais. O Plano Estadual de Gerenciamento Costeiro (Lei No 10.019 de 3 de julho de 1998) divide, em seu terceiro artigo, a zona costeira do estado de São Paulo em quatro setores: Litoral Norte, Litoral Centro, Litoral Sul (Complexo Estuarino-Lagunar de Iguape e Cananéia) e Vale do Ribeira (Figura 1). O Litoral Sul é uma região mais estagnada demograficamente e que conta com atividades de turismo ecológico e agricultura. O Litoral Norte é mais dinâmico em termos demográficos e econômicos, sobretudo com relação às jazidas de petróleo e gás, além da forte presença da atividade turística devido às suas belezas naturais. O Litoral Centro (Baixada Santista) possuía cerca de 1,7 milhões de habitantes em 2017, de acordo com o Instituto Brasileiro de Geografia e Estatística (IBGE), número que pode triplicar em períodos de pico turístico sazonal (Zündt, 2006). Este setor do litoral de São Paulo possui áreas com intensa urbanização, exploração do turismo, atividades industriais em larga escala e, também, ecossistemas complexos de extrema importância ambiental. Em Santos está situado o maior complexo portuário da América do Sul, gerador de emprego e renda, e que escoa grande parte da produção industrial e agrícola do Brasil para os mercados internacionais, movimentando cerca de 80 milhões de cargas ao ano e gerando 25\% da balança comercial do Brasil (São Paulo, 2013). O Vale do Ribeira concentra atividades de agricultura e pecuária, além de biodiversidade pouco explorada (França, 2005).

A problemática da mitigação e da adaptação às mudanças climáticas tem sido abordada, por parte da literatura acadêmica, de forma mais consistente no que diz respeito às suas dimensões urbanístico-demográficas (e.g. Marandola Junior et al., 2013), ecossistêmicas (e.g. Nobre et al., 2010), e institucionais - com foco no que o poder público deve fazer, em decorrência de recomendações de cientistas (e.g. Egler \& Gusmão, 2014) -, e menos em entender como os gestores ambientais percebem o problema e como efetivamente funcionam os processos de tomada de decisão ambiental (cf. Runhaar et al., 2012). A pesquisa aqui reportada buscou colaborar com o debate acadêmico através da documentação das percepções de gestores ambientais da região costeira do estado de São Paulo - em cargos eletivos ou não - a respeito das mudanças climáticas, o tipo e o nível de conhecimento que têm sobre o assunto, bem como documentar em que medida atividades de mitigação e de adaptação por parte das agências e organizações nas quais tais gestores atuam estão sendo criadas e colocadas em prática.

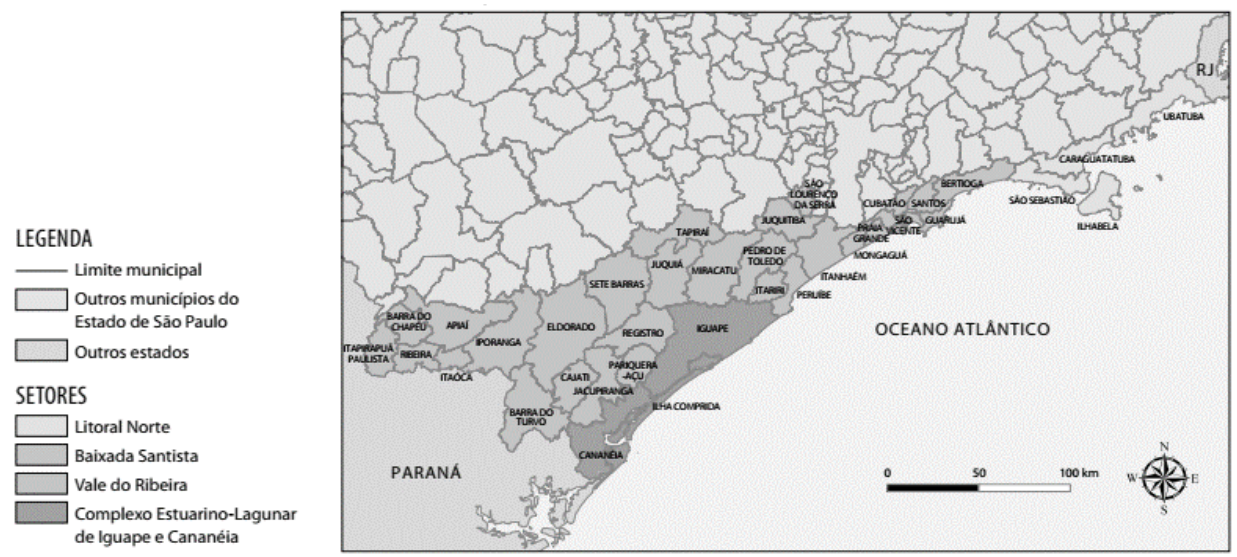

Figura 1. Divisão setorial da zona costeira do estado de São Paulo. Fonte: SÃO PAULO, 2013.

\section{FUNDAMENTAÇÃO TEÓRICA}

Há consenso científico de que está havendo mudança de natureza antrópica no clima global, com o aumento da temperatura da superfície do planeta, o derretimento de geleiras, o aumento do nível do mar e a intensificação da variabilidade climática (Frumkin et al., 2008). 
As mudanças climáticas são definidas como alterações no estado do clima que podem ser identificadas através de dados estatísticos, pela alteração na média ou na variabilidade de suas propriedades, durante uma década ou mais (Edenhofer \& Madruga, 2012), sendo a origem dessas mudanças de causa natural ou antrópicas (Intergovernmental Panel on Climate Change, 2007). As alterações climáticas vêm acompanhadas de uma série de consequências, tais como alterações no regime de chuvas, problemas no abastecimento de água, tempestades, enchentes, deslizamento de encostas, invasão do mar em cidades costeiras, entre outros, o que, por sua vez, exacerba problemas socioeconômicos (Taddei, 2016). O aumento das temperaturas médias, a elevação da temperatura na superfície do mar e o crescimento da frequência de condições meteorológicas extremas trazem às nações complexos problemas logísticos, sociais e políticos (UNESCO, 2013). Também é esperado o aumento nas taxas de morte por doenças cardiorrespiratórias diretamente relacionadas com a mudança do clima e a alteração na distribuição espacial de alguns vetores de doenças infecciosas (Confalonieri \& Marinho, 2007).

No Brasil, como nas demais regiões do planeta, a situação das regiões costeiras, no que diz respeito ao novo panorama climático, é preocupante. Os efeitos mais alarmantes dessas mudanças são o aumento do nível dos oceanos e a maior ocorrência de eventos extremos na zona costeira, como chuvas intensas, tempestades tropicais e fenômenos relacionados às ressacas marítimas (Carmo et al., 2012). As áreas costeiras são diretamente suscetíveis ao aumento do nível do mar, o que pode causar inundações litorâneas, alterações no sistema de ressurgência costeira e intrusão da água do mar em lençóis aquíferos (Campos, 2008). A zona costeira abriga a interface entre atmosfera, continente e oceano, sendo, portanto, vulnerável nas três frentes, onde sofre os efeitos das mudanças climáticas através do aumento do nível médio do mar, aumento dos extremos do clima, secas prolongadas, tempestades mais intensas e possíveis mudanças na propagação das ondas decorrentes de uma nova configuração da circulação atmosférica (Neves \& Muehe, 2008).

Muitos dos problemas gerados pelas alterações climáticas afetarão, sobretudo, as populações que se encontram em situação vulnerável. Moradias desestruturadas e em locais de risco, problemas de infraestrutura e saneamento, fragilidade no setor da saúde são fatores que produzem vulnerabilidade social (Di Giulio \& Ferreira, 2013, 2014). A vulnerabilidade está ligada aos tipos de risco e depende de fatores sociais que podem contribuir ou não para o aumento das fragilidades dessas populações, de modo que pessoas vivendo em uma mesma localidade podem ser afetadas distintamente pelo mesmo evento (Cunha, 2006). Estudos mostram, por exemplo, que mulheres são mais vulneráveis que homens aos impactos das mudanças climáticas (Global Gender and Climate Alliance, 2013; Taddei \& Gamboggi, 2009).

A vulnerabilidade pode se desdobrar em três dimensões: a física, a gerencial e a social (Carmo et al., 2012). A vulnerabilidade física se refere à possibilidade de colapso de serviços e infraestrutura diante dos eventos extremos advindos das mudanças climáticas. A gerencial é a que diz respeito à falta de preparo dos gestores para lidar com as incertezas que envolvem as mudanças e as possíveis formas de intervenção. Por fim, a vulnerabilidade social é aquela relacionada à exclusão de determinados grupos a serviços sociais básicos. A vulnerabilidade de um sistema é seu grau de suscetibilidade e condição de instabilidade diante de algum risco. Nas sociedades democráticas modernas, a elaboração de políticas públicas adquire formato de negociação entre comunidades e agente econômicos. No contexto das mudanças climáticas, isso assume um modelo de gestão englobando vários setores, como saúde, desenvolvimento urbano, industrial, habitação, em distintas escalas espaciais e temporais (Egler \& Gusmão, 2014). Acselrad (2006), por sua vez, sugere que a vulnerabilidade seja tratada através de seus vieses processual e relacional, abordagem que aproxima os contextos locais e as populações afetadas do trabalho analítico.

Uma das linhas de análise das desigualdades ambientais diz que estas são fortemente afetadas pelo mercado de terras, uma vez este induz minorias e famílias de baixa renda a se instalarem em locais onde o risco já era existente (Napton \& Day, 1992). A elevação do nível do mar deixará populações que residem na região costeira mais exposta aos riscos de inundações, erosão, tempestades e outros perigos, podendo provocar deslocamento de grandes contingentes populacionais, o que, juntamente com o abandono das áreas mais atingidas por secas e desertificação, irá gerar nos próximos trinta anos o fenômeno que tem 
sido chamado de "refugiados do clima", aumentando a pressão por moradia e outros serviços básicos nas regiões de destino (Biermann \& Boas, 2010; Vargas, 2011). Logo, o cenário previsto exige preparo adequado de gestores e elaboradores de políticas públicas para lidar com as consequências, não só após sua ocorrência, mas também por meio de medidas adaptativas e preventivas.

As pesquisas científicas a respeito do panorama climático mundial alçam a necessidade de adaptação à condição de imperativo. O quinto relatório do Intergovernmental Panel on Climate Change (Intergovernmental Panel on Climate Change, 2014) aponta que, no cenário mais otimista, a temperatura média subiria entre 0,3 e 1,7 graus e o nível do mar 0,26 a 0,59 metros acima do atual, até o final do século XXI. No pior cenário, a superfície da Terra poderá aquecer entre 2,6 e 4,8 graus ao longo do século, além de ter o nível dos oceanos elevado entre 0,45 e 0,82 metros. Estimativas da última década do século XX, feitas por satélite de altimetria, sugerem que o aumento foi de cerca de três milímetros por ano (Cabanes et al., 2001). O reconhecimento de que a mudança climática é um problema global, mas que os impactos serão sentidos de forma aguda em nível local, é necessário para que o devido foco seja colocado na realização de diagnósticos em escala regional e também a fim de atentar para o fato de que a percepção dos riscos associados às mudanças climáticas está relacionada a atividades sociopolíticas locais (Aslam, 2013).

As ações de mitigação e adaptação têm como objetivo minimizar os impactos negativos das mudanças climáticas ou prevenir a ocorrência delas. Sua execução está condicionada às atividades de diagnóstico do problema e planejamento de ações por parte dos gestores regionais. Dentro do modelo institucional vigente de organização das formas de interação do poder público com os ecossistemas, cabe às agências ambientais e seus colegiados associados a gestão dos territórios que se fazem cenário das mudanças climáticas. Tais agências e colegiados devem estar preparados para lidar com os efeitos destas alterações ecossistêmicas e minimizá-los. Neste contexto, é relevante entender como os responsáveis pelo planejamento local entendem as mudanças climáticas e como isso se relaciona com suas atividades de gestão ambiental (Aslam, 2013; Centro de Pesquisas Sobre Decisões Ambientais, 2016).

Como fartamente documentado na bibliografia, a fronteira entre ciência e política é complexa e em muitas situações se constitui em obstáculo na ligação entre a informação de caráter técnico-científico e os processos de tomada de decisão (Cash et al., 2002; Taddei, 2017). Em um contexto de alterações ambientais, é relevante compreender as ferramentas e estratégias, materiais e cognitivas, usadas por distintas sociedades para lidar com as incertezas ambientais (Di Giulio et al., 2017); os estudos empíricos provam o quanto os sistemas de conhecimento e as instituições políticas são falhos na tentativa de prever os processos naturais e obter seu controle (Taddei, 2012; Taddei \& Gamboggi, 2010). A dificuldade em associar conhecimento e ação pode implicar na não efetividade das decisões tomadas. Os relatórios do IPCC trazem diversos estudos sobre impactos e possíveis adaptações necessárias frente às mudanças no clima; entretanto, há uma insuficiência de pesquisas focando no Brasil, bem como no estado de São Paulo e sua região costeira (Lucon et al., 2009). Entender como os gestores com poder de decisão utilizam as informações que têm a respeito do assunto para planejar as adaptações necessárias é um passo importante para o estabelecimento das políticas públicas de mitigação e adaptação na região costeira, face aos transtornos climáticos. Compreender como as decisões são tomadas torna possível a implementação de políticas para mitigar efetivamente as consequências negativas dos fenômenos relacionados ao clima (Lemos, 2003). Melhores percepção e compreensão públicas sobre as mudanças climáticas podem contribuir para discussões políticas mais informadas e científicas sobre o assunto (Bord et al, 1998).

\section{METODOLOGIA}

Esta pesquisa foi realizada através de um questionário contendo cinquenta e nove questões, aplicadas pela plataforma online SurveyGizmo, num período de quatro meses (setembro a novembro de 2014). O questionário foi dividido em quatro partes: desafios atuais 
da gestão costeira; a gestão costeira e as mudanças climáticas; identificação, de necessidades de informações, ferramentas e treinamentos; e informações sobre o perfil do participante.

A pesquisa baseou-se em questionário utilizado na pesquisa "Rising to the challenge: Results of the 2011 California coastal adaptation needs assessment" realizada, com a mesma finalidade, por Hart. et al (2012), na Califórnia, Estados Unidos. As perguntas foram traduzidas e adaptadas à realidade da região em estudo.

Os participantes foram selecionados em função de sua atuação na gestão costeira do Estado de São Paulo, abrangendo profissionais do setor público (agências e autarquias ligadas à gestão ambiental) das três jurisdições (municipal, estadual e federal), que atuam nos municípios costeiros, e membros de órgãos colegiados ligados à gestão ambiental na região (comitês de gestão de áreas de proteção ambiental e comitês de bacias hidrográficas; incluem-se em tais colegiados profissionais do setor privado). Os contatos foram obtidos junto à direção das agências e órgãos colegiados. A circunscrição dos participantes foi definida pelo Zoneamento Econômico Ecológico (ZEE) da Baixada Santista, que foi o foco desta pesquisa. Contudo, devido a integração de ações de atores dos quatro setores regionais, foram obtidos os contatos de gestores ambientais do Litoral Norte, Litoral Sul e Vale do Ribeira. Apesar de a pesquisa englobar gestores das quatro regiões, os dados mais representativos são os da Baixada Santista.

A solicitação de participação na pesquisa foi feita através de ofícios enviados diretamente aos órgãos e, também, contato direto com os gestores em reuniões de comitês participativos ou em entrevistas pessoais. Após a seleção dos potenciais participantes, o link que dava acesso ao questionário foi enviado para aproximadamente 500 contatos via e-mail. Cerca de metade dos convites foram enviados pelas próprias instituições, quando restrições regimentais impediam a distribuição de listas de e-mails para terceiros.

Foram registrados 179 acessos ao questionário, que foi respondido completamente por $38,5 \%$ participantes. A pesquisa contou com o consentimento informado dos participantes, tendo estes a liberdade de não responder a uma ou mais perguntas. Em razão disso, o número de respostas pode variar de uma pergunta para outra. As respostas foram computadas pela própria plataforma de dados (SurveyGizmo), que gerou gráficos e estatísticas de todas as respostas ${ }^{2}$. Este material serviu de base para a apresentação dos resultados.

\section{RESULTADOS E DISCUSSÃO}

Os dados obtidos revelam que os gestores ambientais que participaram dessa pesquisa atuam majoritariamente na região da Baixada Santista, como mostrado na Figura 2. Além disso, $82,3 \%$ dos respondentes ocupam cargos não eletivos e os demais $(17,7 \%)$ possuem cargos eletivos, atuando, em sua maioria, em órgãos públicos $(75,4 \%)$. Estes cargos são predominantemente de jurisdição estadual, municipal ou regional (75,5\%), sendo o restante pertencente a gestores federais (Figura 3). Aproximadamente 23,7\% dos participantes dedicam mais de $65 \%$ do seu tempo a questões ligadas à gestão ambiental. No geral, os gestores ambientais que participaram da pesquisa possuem alta escolaridade, como ensino superior completo (37,3\%), mestrado (35,6\%) e doutorado (20,3\%). O alto nível de instrução indica que os cargos são predominantemente ocupados por pessoas tecnicamente capazes de elaborar medidas de redução de danos para lidar com os desdobramentos das mudanças climáticas. Os cargos ocupados há mais de dez anos somam 59,3\% dos casos, o que sugere o preenchimento de cargos por autarquia.

\footnotetext{
2 Os dados tabulados de todas as perguntas do questionário estão disponíveis na internet, no link https://umaincertaantropologia.org/resultados-da-pesquisa-atitudes-e-percepcoes-de-gestores-ambientais-da-zonacosteira-de-sp-frente-as-mudancas-climaticas/
} 


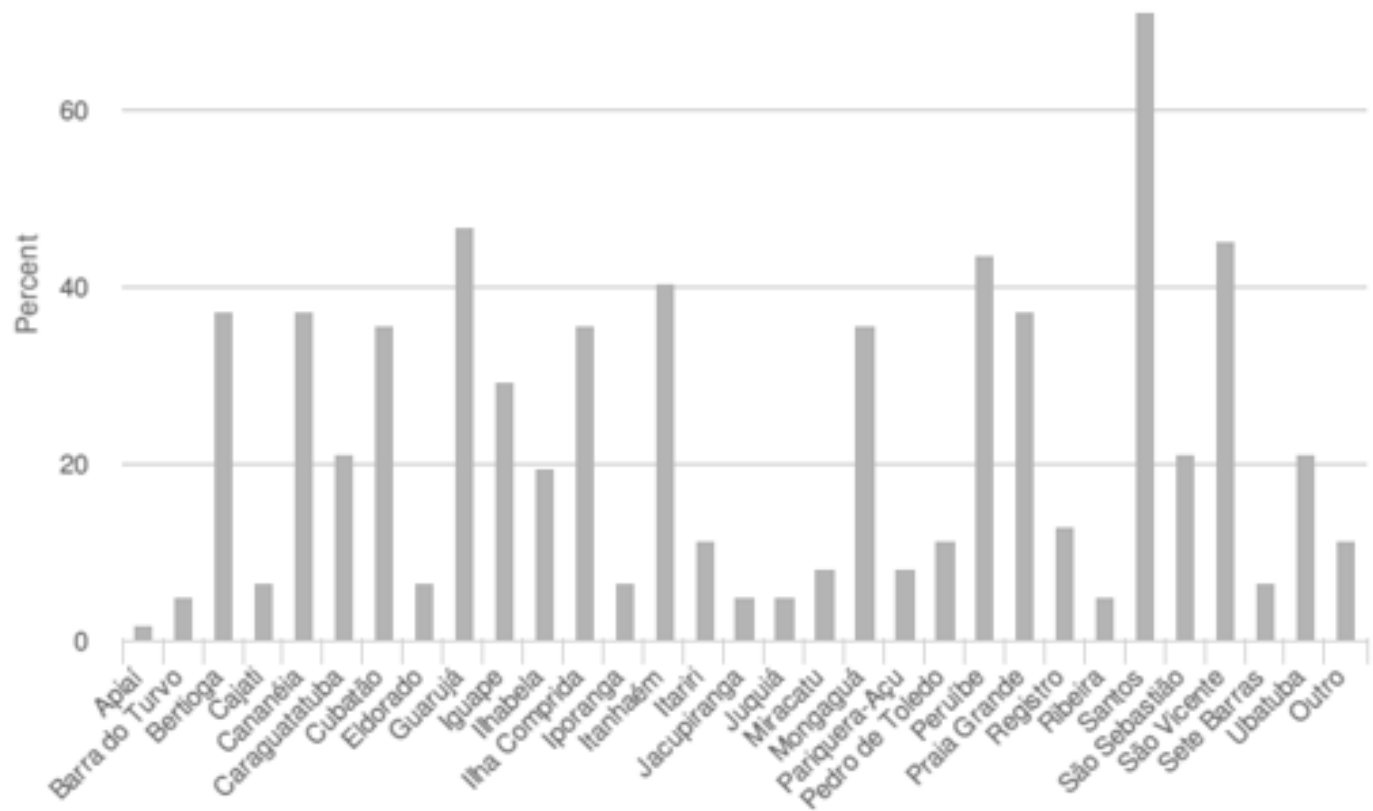

Figura 2. Municípios em que atuam os gestores ambientais da zona costeira de São Paulo. Como a maioria atua em mais de um município, a somatória dos percentuais não totaliza $100 \%$.

Uma das características da zona costeira do estado de São Paulo é a presença de infraestruturas e habitat naturais suscetíveis a variações ambientais, sendo que foram consideradas as mais suscetíveis aos impactos das mudanças climáticas o mangue $(85,6 \%)$, áreas de desenvolvimento turístico $(68,8 \%)$, áreas de desenvolvimento demográfico $(66,4 \%)$, portos e estaleiros $(63,2 \%)$, marinas e estruturas ligadas à pesca esportiva (57,6\%), áreas poluídas ou contaminadas (55,2\%), imóveis residenciais (54,4\%), habitat de espécies ameaçadas (53,6\%), estruturas de saneamento básico (52\%), estruturas ligadas à pesca comercial $(45,6 \%)$, locais históricos e monumentos $(45,6 \%)$, praias desertas ou recreativas $(41,6 \%)$, estradas ou rodovias $(38,4 \%)$, estruturas de tratamento de água $(31,2 \%)$, espaço abertos e parque recreativos $(30,4 \%)$, estruturas de proteção contra a erosão das praias $(26,4 \%)$ e estruturas de proteção contra inundação $(15,2 \%)$. Adicionalmente, $53,1 \%$ dos participantes classificaram que há desenvolvimento significativo ou extremo destas infraestruturas e que esse desenvolvimento está ocorrendo principalmente na orla marinha $(59,2 \%)$ e nas regiões internas dos municípios (52\%).

A partir de uma lista de desafios de gestão, os participantes indicaram os mais preocupantes, sendo o primeiro a perda de habitat naturais, especialmente o mangue, e de espécies protegidas (18,2\%); seguido de expansão urbana desordenada (16,8\%); infraestrutura urbana inadequada, incluindo saneamento básico (12\%); a eliminação de resíduos nas praias, estuário e mangues $(8,8 \%)$; e conflito de usos de recursos naturais $(7,2 \%)$. Ainda, aproximadamente $45 \%$ consideram esses desafios de gestão conflituosos, enquanto $39,8 \%$ classificaram tais desafios como altamente conflituosos. $57,1 \%$ classificaram que houve aumento nas dificuldades de atuação nos últimos cinco anos. Ao mesmo tempo, aproximadamente $55 \%$ creem que as dificuldades de atuação sobre esses desafios de gestão mencionados diminuirão ou permanecerão como estão. 


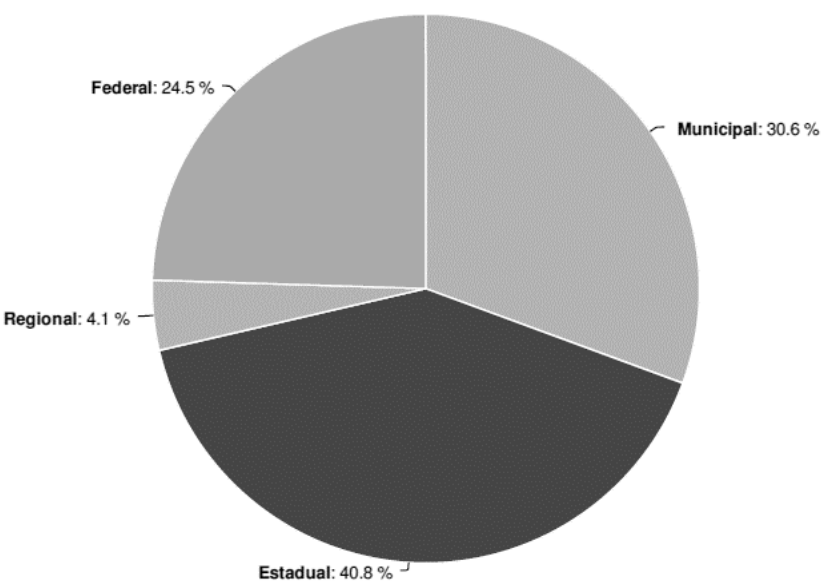

Figura 3. Jurisdição de atuação dos gestores ambientais da zona costeira do estado de São Paulo participantes da pesquisa.

Os participantes também classificaram que os desafios de gestão atual são de responsabilidade, principalmente, dos governos municipais $(71,4 \%)$, das agências estaduais $(55,1 \%)$ e agências ou departamentos federais (38,8\%). Tais dados evidenciam que a gestão ambiental esbarra nos problemas de fragmentação intersetorial, em que a União transfere encargos para estados e municípios e também para organizações não governamentais e a iniciativa privada. Contudo, essa medida se torna ineficiente quando não acompanhada de recursos ou vias de implementação das políticas (Lima, 2011).

Segundo a pesquisa, aproximadamente $91 \%$ dos gestores ambientais se mostram preocupados em algum nível com as mudanças climáticas (Figura 4). Adicionalmente, cerca de $95 \%$ dos respondentes afirma já ter pensado sobre os potenciais impactos das mudanças climáticas sobre sua área de atuação. $80 \%$ das respostas afirmam que as mudanças já estão acontecendo e seus impactos podem ser sentidos, ao passo que 12,9\% acreditam na ocorrência das mudanças climáticas, mas não esperam ver seus impactos antes de dez ou vinte anos. Outra informação relevante diz respeito a quanto tempo tais gestores vêm refletindo sobre as mudanças climáticas em seu trabalho: $80,2 \%$ afirmam pensar neste assunto há mais de três anos, 10,2\% consideraram o assunto entre um e três anos, $2,4 \%$ trabalham com esse assunto há menos de um ano e $7 \%$ ainda não dedicou tempo a isso.

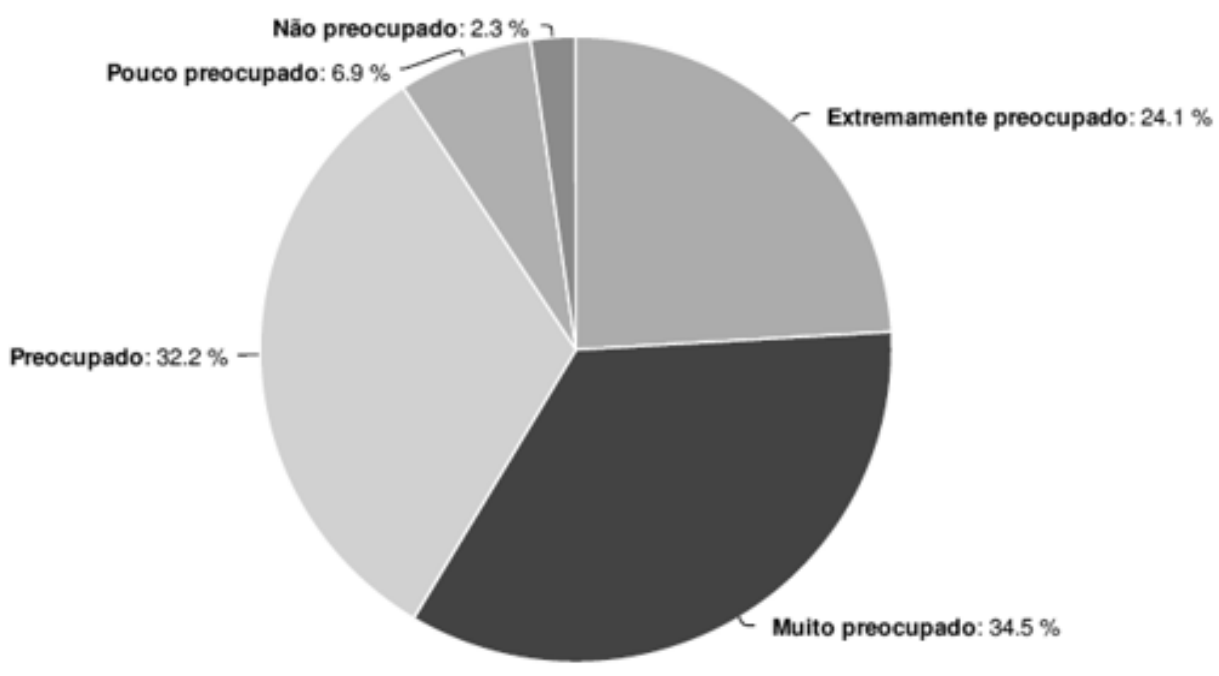

Figura 4. Nível de preocupação dos gestores ambientais em relação a ocorrência das mudanças climáticas. 
Ainda, 93\% dos gestores que participaram da pesquisa acreditam na necessidade de se preparar para os possíveis cenários e efeitos das mudanças climáticas. Destes, $62,8 \%$ dizem que a preparação deve ser baseada nas melhores informações disponíveis a respeito do tema. 3,5\% creem que as informações presentes ainda são insuficientes para a tomada de decisão, bem como alterações em sua forma de atuação.

O questionário também abordou os aspectos de formação e treinamento dos profissionais envolvidos na formulação de políticas de mitigação dos danos e adaptação, e as respostas indicam que 73\% dos participantes não passaram por treinamento formal sobre 0 assunto. Quanto às formas de acesso ao conhecimento e informação, o treinamento pessoal foi considerado extremamente útil por $66,1 \%$ dos gestores, que também ressaltaram a extrema utilidade do nível universitário $(51,7 \%)$, assim como a divulgação de manuais gratuitos $(41,9 \%)$ e a partilha de conhecimento e informação no local de trabalho $(50,8 \%)$. Estudos de caso online foram considerados muito importantes por 51,6\%. Estes números indicam que o grupo envolvido na elaboração de estratégias de mitigação dos danos, derivados das mudanças climáticas, consideram fundamental o preparo da equipe por meio de divulgação do conhecimento e treinamento acerca do tema. A literatura, no entanto, indica que, apesar do entendimento de que a informação é a base de uma gestão eficaz, ela por si só não é capaz de induzir à tomada de decisão por parte dos órgãos gestores (Taddei \& Gamboggi, 2011).

A fonte de informação e de dados mais frequentemente utilizada pela maioria é a internet, ainda que não tenham sido explicitadas as fontes consultadas. Aproximadamente $48 \%$ das respostas indicam a consulta frequente às agências estaduais e federais e $50 \%$ aos colegas de trabalho. A procura por especialistas em instituições de pesquisa é realizada ocasionalmente por $45 \%$ dos respondentes. Revistas profissionais e científicas são consultadas ocasionalmente por, em média, $34 \%$ deles. Nota-se que as fontes mais precisas e confiáveis para fomentar o enfrentamento das mudanças climáticas estão concentradas nas pesquisas e estudos científicos, que os gestores alegam usar com menor frequência. No trabalho dos gestores, são consultados regularmente pela maioria os seguintes dados: planos e mapeamento de uso do solo $(60,3 \%)$; estudos ou mapeamentos de cobertura de solos e habitats (59,1\%); avaliação biológica (59,1\%); mapeamento de risco de inundações e/ou informações sobre a frequência de chuvas (43,9\%); informações sobre clima e tempo (71,6\%); informações sobre marés $(61,2 \%)$; mapeamentos topográficos $(58,3 \%)$.

Os respondentes também consideram ser de alta prioridade o enfrentamento das mudanças climáticas a partir do gerenciamento dos resíduos sólidos $(75,6 \%)$, gerenciamento de estruturas costeiras e urbanas $(68,3 \%)$, planejamento e preparação para os impactos previstos $(64,6 \%)$, criação de áreas protegidas $(61,7 \%)$ e redução das emissões de gases estufa $(49,4 \%)$, sendo os principais motivos que levaram os participantes a se envolver em atividades de planejamento para a adaptação às mudanças climáticas a motivação pessoal $(42,7 \%)$, informação regional ou específica da localidade, mostrando impactos possíveis $(29,4 \%)$ e eventos recentes (26,5\%), a Política Estadual de Mudanças Climáticas (Lei 13.298/2009; 17,7\%), demanda da comunidade $(13,2 \%)$, mandato legislativo municipal $(4,4 \%)$, mandato legislativo estadual ou federal $(4,4 \%)$, ordens de superiores hierárquicos $(4,4 \%)$ e disponibilidade de verbas $(4,4 \%)$.

As condições ambientais são diretamente dependentes das medidas de alguns parâmetros físicos, químicos e biológicos. A alteração destes parâmetros também é um indicativo das mudanças climáticas. As mudanças climáticas podem afetar as condições médias locais e ambiente natural da zona costeira, por isso os respondentes acreditam que nos próximos trinta ou quarenta anos sofrerão aumento da temperatura do ar $(92,9 \%)$, do mar $(80,5 \%)$ e das correntes marinhas $(74,4 \%)$; frequência de inundações $(72,3 \%)$ e tempestades $(78,3 \%)$, intensidade de inundações $(78,6 \%)$ e tempestades $(79,5 \%)$; perturbação de espécies terrestres $(78,3 \%)$ e marinhas $(78,3 \%)$; aumento do nível do mar $(77,4 \%)$ e multiplicação excessiva de algas nos mares $(51,2 \%)$. Por outro lado, os participantes acreditam que ocorrerá uma diminuição na disponibilidade de água (81\%) e na qualidade das águas costeiras $(75,9 \%)$. Em relação à quantidade de escoamento superficial, 37,8\% acreditam que vai ocorrer aumento e os mesmos 37,8\% acreditam na diminuição; $22 \%$ não sabem relacionar tal fato com os efeitos das variações do clima. 
Quando abordados sobre qual era a definição de mudanças climáticas, os participantes discorreram em questão aberta. A maior parte $(62,4 \%)$ das respostas relacionava as alterações no clima à modificação do regime de chuvas, aumento da temperatura média do planeta, elevação do nível do mar, variações climáticas nos níveis global e regional, mudanças nos padrões climáticos, alterações na circulação dos ventos, entre outras alterações físicas associadas ao desequilíbrio do meio e seus componentes.

$67,1 \%$ se consideram razoavelmente informados sobre as questões climáticas, enquanto $24,7 \%$ se consideram bem informados. Porém, apenas $5 \%$ das respostas dadas à questão que solicitava a definição de mudanças climáticas as relacionava a eventos extremos.

De forma geral, no que diz respeito aos órgãos governamentais, há pouco empenho na implementação de projetos de adaptação às mudanças climáticas e mitigação dos danos na opinião de $50 \%$ dos participantes e desinteresse sobre o assunto para $36,1 \%$ deles. 0 baixo empenho na implementação de projetos está claro para $45,4 \%$ dos participantes e para $26,7 \%$ é visível o desinteresse sobre o assunto. Ainda que a opinião dos gestores, expressa nos dados, demonstre o predomínio da preocupação a respeito do clima, ficou evidente que não há relação direta entre o entendimento da questão e a movimentação para que medidas efetivas sejam tomadas. A movimentação dos gestores sobre os efeitos das mudanças climáticas é vista, mas em um cenário de informações insuficientes sobre o assunto para $44,7 \%$, enquanto $41,2 \%$ não percebem nenhum tipo de movimentação nesse sentido.

Dos envolvidos em atividades de planejamento para adaptações às mudanças, 29,4\% dizem ser motivados por informação sobre os prováveis impactos em sua localidade de atuação. Os motivados pela ocorrência de eventos extremos somam 26,5\%. Apenas 17,7\% alegaram motivação a partir do conhecimento da Política Estadual de Mudanças Climáticas, importante instrumento e fonte de informação sobre o assunto.

Neste contexto, a Figura 5 demonstra em qual estágio de ações os gestores participantes da pesquisa se encontravam. As ações relacionadas a favor da mitigação dos danos ainda não começaram em $34,3 \%$ dos casos. Em fase de estudo estão $25,7 \%$ dos casos, $15,7 \%$ em fase de planejamento e $24,3 \%$ em fase de implementação. Os obstáculos identificados pelos participantes para a implementação de medidas para enfrentar os prováveis impactos das mudanças climáticas foram a falta de financiamento para preparar $(60,6 \%)$ e implementar $(69,7 \%)$ um plano de ação; falta de recursos humanos para analisar as informações relevantes (65,2\%); falta de liderança política (59,1\%). A falta de acesso às informações necessárias e de assistência técnica de agências estaduais ou federais é um grande obstáculo para 50\% dos participantes, em média.

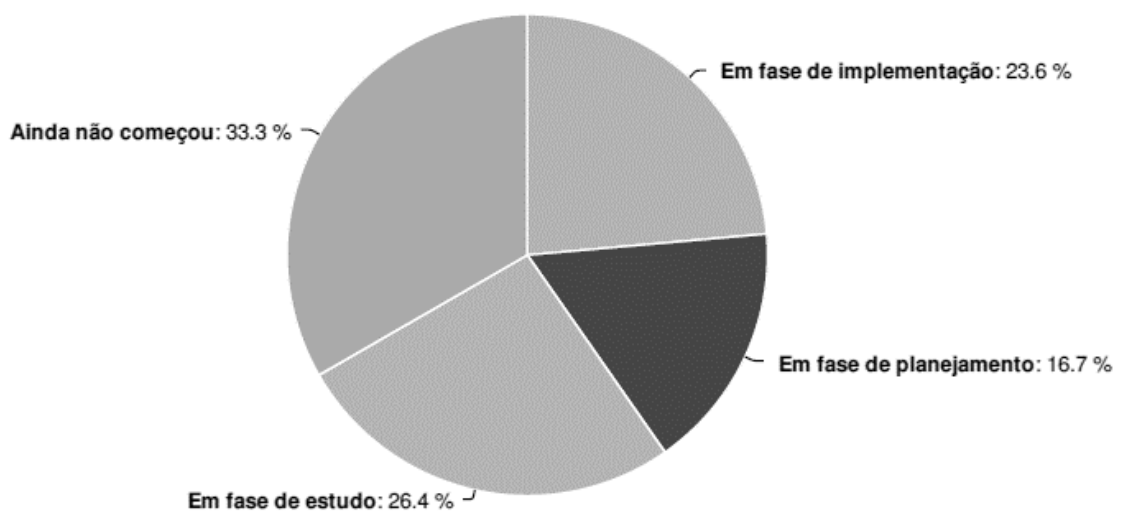

Figura 5. Estágio referente às ações em relação aos impactos das mudanças climáticas.

\section{DISCUSSÃO}

O resultado mais sobressalente dentro do conjunto de dados gerados pela pesquisa é o que mostra que ao mesmo tempo em que os gestores ambientais mostram-se, em sua imensa maioria $(90,8 \%)$, preocupados com as mudanças climáticas e seus impactos previstos, 
em igual montante afirmam testemunhar a inoperância do poder público quanto a essa questão: $15.4 \%$ afirmam que "não há movimentação sobre o assunto" em órgãos governamentais, $44.2 \%$ disseram perceber "falta de empenho", e 34.6\% mencionaram "desinteresse". Tais números variam minimamente quando a questão se refere a instituições privadas, ou seja, os gestores entrevistados percebem-se em situação de dissonância cognitivo-institucional, em que suas percepções do risco envolvido contrastam com a forma como veem as instituições (não) reagirem à gravidade do problema. Claramente não se trata, desta forma, de que os gestores não deem à questão climática o devido valor; mas de que as dinâmicas institucionais não se movem em sincronia com a percepção dos seus técnicos e gestores, de maneira geral. Muitas vezes tal falta de sincronia é implícita, faz parte da cultura das instituições. Mas há situações em que ela se faz explicitada. Ao serem perguntados sobre "como as mudanças climáticas podem afetar o seu trabalho", por exemplo, 17.2\% afirmaram: "eu entendo como tais mudanças vão impactar o meu trabalho, mas eu ainda não tenho autorização para trabalhar em estratégias de adaptação".

Fazendo um paralelo entre o domínio do poder público sobre a organização da gestão ambiental e os aspectos mais gerais das políticas públicas no Brasil, esse resultado não é exatamente surpreendente. O setor público ambiental no país se mostra sistematicamente reativo, promovendo ações de remediação de danos após eventos que impactam negativamente o meio ambiente. Nos casos de inundação, por exemplo, a agenda do poder público local é estruturada ao redor da promoção de ações de realocação de pessoas, reconstrução de casas e a recomposição da região afetada. A postura proativa, focada na prevenção dos efeitos por meio de planejamento e ação de planos de contenção de danos é praticamente inexistente no contexto da gestão ambiental brasileira - e isso ocorre, paradoxalmente, a despeito do protagonismo do estado de São Paulo no que diz respeito à construção de ferramentas de governança ligadas às mudanças climáticas (ver Di Giulio \& Ferreira, 2014; Ramires, 2014). É característica das próprias administrações públicas a adoção de postura permissiva em situações de vulnerabilidade em seu território, como ocupação desordenada e expansão urbana em áreas de risco, para posteriormente (tentar) criar medidas emergenciais para contornar os efeitos decorrentes de tais políticas. De forma aproximada e tomando as diferenças de contexto em conta, a literatura sugere que o fenômeno não se restringe ao litoral do Estado de São Paulo (ver Egler \& Gusmão, 2014 para uma análise da situação do Estado do Rio de Janeiro) e nem ao Brasil (ver Runhaar et al., 2012 e Mulder et al., 2009 para a Holanda; Demeritt \& Langdon, 2004 e Tompkins et al., 2010 para o Reino Unido; ver também Bulkeley, 2010; Wheeler, 2008; e Bassett \& Shandas, 2010). Ocorre, no entanto, que os impactos previstos para os cenários de mudança climática utilizados pelo IPCC sugerem efeitos imensamente mais intensos e frequentes. Em tal situação, a tradicional postura reativa das instituições de gestão ambiental mostra-se, no mínimo, inadequada.

Um outro ponto digno de discussão diz respeito à natureza das mudanças climáticas mencionadas pelos gestores, que decorrências isso tem para o trabalho de mitigação e adaptação. Repetindo dados mencionados acima, chama a atenção que ao mesmo tempo em que $91,8 \%$ dos respondentes disseram que se consideram razoavelmente ou bem informados sobre as questões climáticas, apenas $5 \%$ das respostas dadas à questão aberta que solicitava uma definição de mudanças climáticas as relacionava a eventos extremos. É possível que a falta de ações efetivas de gestão esteja associada à não associação (ou à falta de capacidade de associação) destas aos impactos previstos das mudanças climáticas. Novamente, uma reposta à questão "como as mudanças climáticas podem afetar o seu trabalho" parece corroborar essa hipótese: $23.2 \%$ dos entrevistados responderam: "Estas mudanças podem afetar as coisas que eu gerencio e sobre as quais tomo decisões, mas eu não sei como me preparar para elas". Desta forma, ainda que grande porcentagem dos participantes saiba definir o que são as mudanças climáticas (a dimensão mais abstrata do problema), 95\% dos entrevistados não as associou diretamente com seus efeitos mais catastróficos (a dimensão mais prática) e em nível local, ou seja, dentro do contexto de sua jurisdição enquanto gestor ambiental. Isso sugere que por terem dificuldade em relacionar as dimensões abstrata e prática do problema, a maioria dos gestores não identifica com claridade formas de agir diante desta questão. Como se sabe, os eventos extremos, como inundações e tempestades, 
acompanhados dos eventuais deslizamentos de terra, terão sua frequência e intensidade aumentadas com o aquecimento global, sendo que atualmente estes eventos já são responsáveis por cerca de $72,5 \%$ das vítimas e $75 \%$ das perdas econômicas em termos mundiais (Nunes, 2009). Logo, a elaboração de políticas públicas que desempenhem seu papel de mitigar danos de maneira eficaz exige que os impactos previstos sejam dimensionados da forma mais realista possível, ou seja, localmente contextualizada. Adicionalmente, como sugerem Uittenbroek et al. (2012), as soluções propostas devem estar integradas com políticas públicas já existentes, no que os autores chamam de "convencionalização das mudanças climáticas" (mainstreaming climate change), de modo que tais soluções sejam rapidamente absorvidas pelos processos institucionalizados de elaboração de políticas públicas e transformadas em padrões estabelecidos.

\section{CONCLUSÃO}

Os dados gerados por esta pesquisa mostram que os gestores ambientais atuantes na região da Baixada Santista e demais zonas costeiras do estado estão, em sua maioria, conscientes da existência das mudanças climáticas. Existe o entendimento de que vários dos parâmetros ambientais relevantes às suas atividades profissionais já estão ou serão alterados dentro de alguns anos, modificando os padrões atuais e afetando, de alguma forma, seu trabalho. Os participantes afirmam a necessidade de se preparar, planejando as adaptações necessárias para reduzir impactos e mitigar danos. Apesar disso, há um descompasso entre as preocupações particulares dos respondentes da pesquisa e as ações efetivas de estudo, planejamento e implementação de projetos por parte dos órgãos envolvidos. Mesmo com o entendimento a respeito das mudanças climáticas há falta de preparo sobre como agir diante de tal cenário. Os resultados corroboram os encontrados por outros pesquisadores, recentemente publicados (Barbi et al., 2017; Ferreira et al., 2017).

Existe vasta literatura internacional mostrando que não há correlação direta e imediata entre a disponibilidade de informações - por melhor que sejam - sobre as mudanças climáticas e a mudança de padrões comportamentais (Centro de Pesquisas Sobre Decisões Ambientais, 2016, Taddei \& Gamboggi, 2011). O caso de estudo desta pesquisa fornece evidência nacional nesse sentido: a imensa maioria dos gestores ambientais que participaram da pesquisa têm boas informações sobre as mudanças climáticas, estão preocupados com os impactos previstos, e ainda assim muito pouco efetivamente está sendo feito no sentido de preparar a sociedade e o poder público para tais impactos.

Não é novidade a afirmação da existência de barreiras entre ciência e política, causadoras de dificuldades de comunicação, colaboração, avaliação integrada e tomada de decisão, além da inibição da conexão entre conhecimento e tomada de decisão em situações complexas, tais como as mudanças climáticas (Pfaff et al., 2013). Contudo, muito mais do que uma quebra da barreira entre ciência e política, se faz necessária a criação de plataformas de comunicação que forneçam aos gestores ambientais informações relevantes, confiáveis e legitimas - elementos essenciais para que uma nova informação seja inserida em um contexto decisório (Cash et al, 2002). Nesse sentido, os dados da pesquisa mostram que o formato "científico" da mensagem climática, ainda que essencial para que pesquisadores ao redor do planeta se comuniquem e para que a mensagem possua autoridade junto a tomadores de decisão, por outro lado faz com que ela tenha pouca relevância: os atores locais entendem o conteúdo abstrato, descontextualizado da mensagem, evidenciando seus indicadores físicos em escala global, mas não são capazes de integrá-la em seus processos decisórios, que usam informações em escalas espaciais e temporais distintas, nem de transformar a informação global em previsão em impactos locais contextualizados (como a transformação da ideia genérica de "elevação do nível do mar" para o diagnóstico específico dos impactos na infraestrutura da orla de Santos). Recentemente alguns estudos começaram a ser feitos nesse sentido (Moreira et al., 2017), mas esta transformação se encontra em estágio apenas inicial. 


\section{BIBLIOGRAFIA}

Acselrad, H. (2006). Vulnerabilidade ambiental, processos e relações. Comunicação. Rio de Janeiro: Instituto Brasileiro de Geografia e Estatística (IBGE).

Aslam, S. (2013). Examining the relationship between climate change risk perceptions and the implementation of climate-related policies (Dissertação de mestrado). University of Waterloo, Ontario.

Barbi, F., Ferreira, L. C., \& Joly, C. A. (2017). Mudança climática em cidades da costa brasileira: riscos e respostas políticas em Santos, na Região Metropolitana da Baixada Santista - SP. In L. C. Ferreira, L. Schmid, M. P. Buendía, J. Calvimontes, \& J. E. Viglio. (orgs), Clima de Tensão - Ação humana, biodiversidade e mudanças climáticas (pp. 183-200). Campinas: Editora da UNICAMP.

Bassett, E., \& Shandas, V. (2010). Innovation and climate action planning. Journal of the American Planning Association, 76(4), 435-450.

Biermann, F., \& Boas, I. (2010). Preparing for a Warmer World: Towards a Global Governance System to Protect Climate Refugees. Global Environmental Politics, 10(1), 60-88.

Bord, R. J., Fisher, A., \& O'Connor, R. E. (1998). Public perceptions of global warming: United States and international perspectives. Climate Research, 11(1), 75-84.

Bulkeley, H. (2010). Cities and the governing of climate change. Annual Review of Environment and Resources, 35(1), 229-253.

Cabanes, C., Cazenave, A., \& Le Provost, C. (2001). Sea level rise during past 40 years determined from satellite and in situ observations. Science, 294(5543), 840-842.

Campos, E. (2008). A importância dos oceanos nas mudanças globais. In Relatório da III Conferência Regional de Mudanças Globais (pp 55-59). São Paulo: Instituto de Estudos Avançados da Universidade de São Paulo.

Carmo, R. L., Seixas, S. R. C., \& Vargas, M. C. (2012). Vulnerabilidades às mudanças climáticas no litoral paulista: urbanização, saneamento e saúde. In Anais do VI Encontro Nacional da ANPPAS (p. 18-21). São Paulo: Associação Nacional de Pós-Graduação e Pesquisa em Ambiente e Sociedade.

Cash, D., Clark, W., Alcock, F., Dickson, N., Eckley, N., \& Jager, J. (2002). Salience, Credibility, Legitimacy And Boundaries: Linking Research Assessment And Decision Making. KSG Working Papers Series RWP02-046. http://dx.doi.org/10.2139/ssrn.372280

Centro de Pesquisas Sobre Decisões Ambientais - CRED. (2016). A Comunicação das Mudanças Climáticas: Um guia para cientistas, jornalistas, educadores, políticos e demais interessados. Rio de Janeiro: Centro de Pesquisas sobre Decisões Ambientais.

Confalonieri, U. E. C., \& Marinho, D. P. (2007). Mudança climática global e saúde: perspectivas para o Brasil. Revista Multiciência, 8, 48-64.

Cunha, J. M. P. Novas metrópoles paulistas: população, vulnerabilidade e segregação. Campinas: Universidade Estadual de Campinas, Núcleo do Estudos de População, 2006.

Demeritt, D., \& Langdon, D. (2004). The UK climate change programme and communication with local authorities. Global Environmental Change, 14(4), 325-336.

Di Giulio, G. M., \& Ferreira, L. C. (2014). Contribuições das Ciências Humanas para o debate sobre mudanças ambientais: um olhar sobre São Paulo. Estudos Avançados, 28(82)

Di Giulio, G. M., \& Ferreira, L. C. (2013). Governança do risco: uma proposta para lidar com riscos ambientais no nível local. Desenvolvimento e Meio Ambiente, 28, 29-39.

Di Giulio, G. M., Viglio, J. E., \& Ferreira, L. C. (2017). Alterações climáticas, riscos e percepções: análise sobre uma região costeira do Brasil. In L. C. Ferreira, L. Schmid, M. P. Buendía, J. Calvimontes, \& J. E. Viglio (Orgs.), Clima de Tensão - Ação humana, biodiversidade e mudanças climáticas (pp. 183-200). Campinas: Editora da UNICAMP.

Edenhofer, O., \& Madruga, R. P. (2012). Special Report on Renewable Energy Sources and Climate Change Mitigation. Summmary for Policymakers: A Report of Working Group III of the IPCC and Technical Summary. Intergovernmental Panel on Climate Change.

Egler, C. A. G., \& Gusmão, P. P. (2014). Gestão costeira e adaptação às mudanças climáticas: o caso da Região Metropolitana do Rio de Janeiro, Brasil. Revista da Gestão Costeira Integrada, 14(1), 65-80.

Ferreira, L. C., Barbi, F., \& Giesbrecht, M. (2017). Mudança climática e risco em cidades da costa brasileira: uma análise quali e quantitativa. In: L. C. Ferreira, L. Schmid, M. P. Buendía, J. Calvimontes, \&J. E. Viglio (Orgs.), Clima de Tensão - Ação humana, biodiversidade e mudanças climáticas (pp. 163-181). Campinas: Editora da UNICAMP. 
França, A. M. (2005). Vale do Ribeira (SP): proposições econômicas, sociais, políticas e ambientais para o crescimento e desenvolvimento sustentável dos municípios da Região Administrativa de Registro (Dissertação de mestrado). Economia Política, Pontifícia Universidade Católica de São Paulo, São Paulo.

Frumkin, H., Hess, J., Luber, G., Malilay, J., \& McGeehin, M. (2008). Climate change: the public health response. American Journal of Public Health, 98(3), 435-445.

Global Gender and Climate Alliance - GGCA. (2013). Overview of linkages between gender and climate change. New York: United Nations Development Programme.

Holligan, P., \& Deboois, H. (1993). Land-Ocean Interactions in the Coastal Zone (LOICZ). Science Plan (50 p.). Stockholm: International Geosphere Biosphere Programme, International Council of Scientific Unions.

Intergovernmental Panel on Climate Change - IPCC. (2007). Synthesis Report. Contribution of Working Groups I, II and III to the Fourth Assessment Report of the Intergovernmental Panel on Climate Change. Geneva: IPCC.

Intergovernmental Panel on Climate Change - IPCC. (2014). Synthesis Report. Contribution of Working Groups I, II and III to the Fifth Assessment Report of the Intergovernmental Panel on Climate Change. Geneva: IPCC.

Klein, R. J. T., \& Nicholls, R. J. (1999). Assessment of costal vulnerability to climate change. Ambio, 28(2), 182-187.

Lemos, M. C. M. (2003). A tale of two policies: The politics of climate forecasting and drought relief in Ceara, Brazil. Policy Sciences, 36, 101-123.

Lima, G. F. C. (2011). A institucionalização das políticas e da gestão ambiental no Brasil: avanços, obstáculos e contradições. Desenvolvimento e Meio Ambiente, (23), 121-132.

Lucon, O., Oliveira, F. D. F., \& Alves, J. W. S. (2009). Política Estadual de Mudanças Climáticas (Lei n. 13.798 de 9 de novembro de 2009). - versão zero para consulta pública. São Paulo: Secretaria do Meio Ambiente do Estado de São Paulo - SMA, Companhia Ambiental do Estado de São Paulo - CETESB.

Marandola Junior, E., Marques, C., Paul, L. T., \& Cassaneli, L. B. (2013). Crescimento urbano e áreas de risco no litoral norte de São Paulo. Revista Brasileira de Estudos de População, jan./jun. 30(1), 35-56.

Moreira, F. A., Nunes, L. H., Marengo, J. (2017). Avaliação da capacidade adaptativa (ACI) no município de Santos/SP a partir das mudanças climáticas. Os Desafios da Geografia Física na Fronteira do Conhecimento, 1, 1478-1491.

Mulder, K. F., Enserink, B., \& Salcedo-Rahola, B. (2009, july 5-8). The neglected effects of climate change. In Paper presented at the 15th International Sustainable Development Research Conference. Nederlands: Utrecht University.

Napton, M. L., \& Day, F. A. (1992). Polluted neighborhoods in Texas: Who lives there. Environment and Behavior, 24, 508-526.

Neves, C. F., \& Muehe, D. (2008). Vulnerabilidade, impactos e adaptação a mudanças do clima: A zona costeira. Parcerias Estratégicas, 27, 217-296.

Nobre, C., Young, A. F., Saldiva, P., Marengo, J. A., Nobre, A. D., Alves Júnior, S., Silva, G. C. M., \& Lombardo, M. (2010). Vulnerabilidades das Megacidades Brasileiras às Mudanças Climáticas: Região Metropolitana de São Paulo. Sumário Executivo - junho de 2010. Rio Claro: INPE, IPT, UNICAMP, USP, UNESP. Recuperado em 10 setembro 2013, de http://mudancasclimaticas.cptec.inpe.br/ rmclima/pdfs/publicacoes/2010/SumarioExecutivo_mega cidades.pdf

Nunes, L. H. (2009). Mudanças climáticas, extremos atmosféricos e padrões de risco a desastres hidrometeorológicos. População e mudança climática: dimensões humanas das mudanças ambientais globais (pp. 53-73). Campinas: Nepo/Unicamp; Brasília: UNFPA.

Pfaff, A., Velez, M. A., Taddei, R., \& Broad, K. (2013). Unequal Information, Unequal Allocation: Bargaining field experiments in NE Brazil. Environmental Science \& Policy, 26, 90-101.

Ramires, J. Z. S. (2014, setembro 8-12). A gestão dos riscos climáticos em São Paulo. In Trabalho apresentado no VI Congresso Iberoamericano de Estudios Territoriales y Ambientales, São Paulo: Faculdade de Filosofia, Letras e Ciências Humanas da Universidade de São Paulo.

Runhaar, H., Mees, H., Wardekker, A., Van Der Sluijs, J., \& Driessen, P. P. J. (2012). Adaptation to climate change-related risks in Dutch urban areas: stimuli and barriers. Regional Environmental Change, 12, 777-790. 
Sachs, J. D., Mellinger, A. D., \& Gallup, J. L. (2001). The geography of poverty and wealth. Scientific American, 284(3), 70-75.

São Paulo. Coordenadoria de Planejamento Ambiental. Secretaria do Meio Ambiente do Estado de São Paulo (2013). ZEE - Zoneamento Ecológico-Econômico - Setor Costeiro Da Baixada Santista. L. R. N. Oliveira (Org.), B. S. Caio, C. M. Barros, F. Chapuis, H. S. Carmona, I. L. S. Parada, L. Souza e Silva, M. R. Itani, M. A. O. Gonçalves, \& N. M. Cruz (Equipe técnica). São Paulo: SMA.

Taddei, R. (2017). Meteorologistas e profetas da chuva: conhecimentos, práticas e políticas da atmosfera. São Paulo: Terceiro Nome.

Taddei, R. (2016). Os desastres em uma perspectiva antropológica. ComCiência, 176, 1.

Taddei, R. (2012). The politics of uncertainty and the fate of forecasters. Ethics, Policy \& Environment, 15(2), 252-267.

Taddei, R., \& Gamboggi, A.L. (2011). Etnografia, meio ambiente e comunicação ambiental. Caderno Pedagógico, 8(2), 9-28.

Taddei, R., \& Gamboggi, A. L. (2009). Gender and the semiotics of political visibility in the Brazilian northeast. Social Semiotics, 19, 149-164.

Taddei, R., \& Gamboggi, A. L. (2010). Introdução. In Taddei, R., \& Gamboggi, A. L. (Orgs.). Depois que a chuva não veio. Respostas sociais às secas na Amazônia, no Nordeste e no Sul do Brasil (pp. 7-31). Fortaleza: Fundação Cearense de Meteorologia e Recursos Hídricos/Comitas Institute for Anthropological Study.

Tompkins, E. L., Adger, W. N., Boyd, E., Nicholson-Cole, S., Weatherhead, K., \& Arnell, N. (2010). Observed adaptation to climate change: UK evidence of transition to a well-adapted society. Global Environmental Change, 20(4), 627-635.

Turner, R. K.; Subak, S., \& Adger, W. N. (1996) Pressures, trends, and impacts in coastal zones: Interactions between socioeconomic and natural systems. Environmental Management, 20(2), 159173.

Uittenbroek, C. J., Janssen-Jansen, L. B., \& Runhaar, H. A. C. (2012). Mainstreaming climate adaptation into urban planning: overcoming barriers, seizing opportunities and evaluating the results in two Dutch case studies. Regional Environmental Change, 13, 399-411.

UNESCO. (2013). World Social Science Report. Recuperado em 10 de janeiro de 2014, de http://www.keepeek.com/Digital-Asset-Management/oecd/social-issues-migration-health/worldsocial-science-report-2013_9789264203419-en\#page1.

Vargas, M. C. (2011). Mudança climática e recursos hídricos: problemas de adaptação na escala metropolitana. O caso da região da Baixada Santista, Brasil. In V Congresso Iberoamericano Sobre Desarrollo y Ambiente, Santa Fe. Santa Fe: Facultad de Ingeniería y Ciencias Hídricas de la Universidad Nacional del Litoral.

Wheeler, S. M. (2008) State and municipal climate change plans. Journal of the American Planning Association, 74(4), 481-496.

Zündt, C. (2006). Baixada Santista: uso, expansão e ocupação do solo, estruturação de rede urbana regional e metropolização. Novas metrópoles paulistas: população, vulnerabilidade e segregação. Campinas: Núcleo de Estudos de População/Unicamp.

Submetido 19/Fev./2018.

Aceito 21/Abr./2019

Classificação JEL: Global Warming 